\title{
Studying the Interrelationship amongst various success factors for partnering in Construction Industry in Developing Countries
}

\author{
Remica Aggarwal \\ School of Business \\ University of Petroleum \& Energy \\ Studies \\ Dehradun, India
}

\author{
S. P. Singh \\ Department of Management \\ Studies \\ Indian Institute of Technology \\ Delhi, India
}

\author{
Veena Aggarwal \\ Recventures Education Services \\ Private Limited \\ Delhi, India
}

\begin{abstract}
The paper focuses on exploring the success factors for successful partnering in construction industry projects. Further it studied the interrelationship amongst them using ISM methodology.
\end{abstract}

\section{Keywords}

Partnering ISM methodology, Construction project management

\section{INTRODUCTION}

The construction industry is a typical industry that works in temporary organizations, i.e. projects. It typically has an engineer to order setup where most of the products are physically big and immobile and consequently have to be produced on the spot [1-5]. Materials used in construction industry majorly falls into basic categories such as heavy materials (typically concrete, sand, gravel, bricks, timber, etc.) ; light materials (which include structure completion and decorating materials [6]); standard materials (such as plasterboards or kitchen cabinets [2]) and project specific materials (prefabricated concrete elements and ventilation installations). These materials make up for a large part of the construction cost and a construction project is depending on suppliers and transport providers to deliver materials to them [7]. These material suppliers could be manufacturers and wholesalers or building merchants.

To deal with uncertain market situations and economic cycles; diverse and dynamic client behavior; requirement of specialist manpower; large heavy and immobile products (such as building tunnels, bridges etc. ) require to be built on the construction etc. As much as 60-80\% of the gross work done in construction projects involves the buying-in of materials and services from suppliers and subcontractors, leading to that these supply chain actors heavily impact the performance of construction projects $([2,3],[8])$. Hence, the construction supply chain is regarded as complex with interactions between multiple actors during the construction process [9]. Also construction industry is a large industry sector employing hundreds of thousands and a large contributor to a country's GDP. Therefore the problems have a large societal impact. It is really hard for construction companies to keep pace with the multiple demands of the clients in terms of lower cost, higher quality, shorter execution duration and more reliable schedules. Partnering has been but forward as a solution to overcome the temporariness and the adversarial relationships in the construction.
Partnering as per Construction Industry Institute $[10,11]$ has been cited as a long-term commitment between two or more organizations for the purposes of achieving specific business objectives by maximizing the effectiveness of each participant resource. Concept of partnering was first introduced by USA armed forces in the 1980's to increase efficiency in construction ([12,13]). Since then partnering and other collaborative practices has been used in the construction industry $([14,15])$ and are often associated with and said to generate several advantageous benefits such as reduced costs,improved quality, reduced lead times, increased productivity,increased sustainability, increased number of innovations, etc.([16 -21]). Although partnering exists in many forms but thetwo common forms are project partnering and strategic partnering. ([2,3,22,23]). Project partnering is the most common and popular form of partnering. It suits most kind of projects, and since it is a partnering form that only stretches across one project it is often used in publicly funded construction projects where public procurement acts hinder long term agreements $([12,13,15,23,24])$. In project partnering agreements, trust has been established between the parties and there is also an expected increase in communication and mutual understanding. The parties also share common, project specific goals. This is a real form of partnering but quite short term and therefore offers limited benefits. On the other hand strategic partnering is a more mature, long-term relationship between the parties that stretch over several projects $([12,13,18,24])$. Strategic partnering involves high trust between the parties and the relationship evolves further to include development of products, services, procedures, etc. The parties shall engage in the partnership on equal terms and share both risks and rewards throughout the collaboration.

The objective of the paper is to identify various success factors for partnering and then study the inter-relationship amongst them using ISM methodology. The paper is organized as follows. Section 2 deals with literature review.Section 3 explains the ISM methodology and thereafter it has been explained through case example in section 4. Finally, managerial implications and directions for future research have been discussed in section 5 . 


\section{LITERATURE REVIEW ON PARTNERING AND ITS SUCCESS FACTORS}

Several analysts and writers have identified critically a variety of requirements and factors responsible for the success of partnering relationships in construction industry. Among these analysts are [25-27]. [28] identified the four major factors viz. collaborative team culture ; long-term quality perspective ; consistent objectives and resource sharing. Trust and mutual understanding are necessary and common components and are central to all partnering and outsourcing projects is illustrated by [29]. [30] has elaborated further on how the components can be interpreted and distinguish between "hard" and "soft" components. However, [31] criticized the dichotomization of project management theories into "hard" and "soft" and indicated that components are opposite to each other. Organizational culture as well as national and international culture attribute to varying success of partnering. For example, partnering has been very successful in the UK and south-east Asia and has only in recent years been increasing in the Nordic countries such as Denmark $([12,13,18,23])$. Today most partnering agreements are dyadic, typically only between the client and the main contractor $(23,19,20,32]$. Often the consultants also are included $([23,18])$. Although some suppliers have initiated partnering agreements of their own [33], many suppliers are skeptical about partnering, viewing it as a way to push costs upstream in the supply chain $[23,34]$.

Authors have explored various success factors for partnering which have been cited in the following table . Several researchers have studied, defined and discussed the critical success factors of partnering in construction [27,28,35,36,37]. Critical success factors may be controversial, as they can be affected by local culture and practices. Nevertheless, mutual trust, effective communication, support from top management, clearly defined responsibilities, mutual goals, a dedicated team, commitment to continuous improvement and a win-win attitude are considered to be the most cited critical success factors in these studies. These success factors have been investigated in the context of construction companies and construction owners and are valid in the construction phase of building projects. Table I below shows the various success factors along with their references. Authors have also made use of internet search engines, use of Mendeley software etc. to search for the relevant literature using keywords such as partnering in construction industry ; partnering in India, success factors for partnering, barriers for partnering, partnering amongst contractors and clients etc.

Table I : Success factors for Partnering in Construction industry

\begin{tabular}{|c|c|c|}
\hline 1. & $\begin{array}{c}\text { Effective communication / } \\
\text { clear lines of } \\
\text { communication (CLC) }\end{array}$ & $\begin{array}{c}[27], 35],[36],[37],[38], \\
{[39],[40]}\end{array}$ \\
\hline 2. & $\begin{array}{c}\text { Effective co-ordination (EC) } \\
{[24],[27],[35],[36],} \\
{[37],[38],[39],[40],[41]}\end{array}$ \\
\hline 3. & Resource sharing (RS) & {$[24],[28],[41]$} \\
\hline 4. & $\begin{array}{c}\text { Dedicated team culture } \\
\text { (DTC) }\end{array}$ & {$[24],[41]$} \\
\hline 5. & $\begin{array}{c}\text { Long term relationships } \\
(\text { LTR) }\end{array}$ & {$[19],[20],[39],[24]$,} \\
& \multicolumn{2}{|c|}{$[28],[41]$} \\
\hline
\end{tabular}

\begin{tabular}{|c|c|c|}
\hline 6. & $\begin{array}{c}\text { Mutual objectives/ goals } \\
\text { and mutual trust (MO/MT) }\end{array}$ & $\begin{array}{l}{[26],[28],[35],[36],[37],} \\
{[38],[39],[40],[27],[42]}\end{array}$ \\
\hline 7. & $\begin{array}{c}\text { Management support (MS) } \\
\text { and financial security }\end{array}$ & {$[26],[40][24],[41]$} \\
\hline 8. & $\begin{array}{l}\text { Dynamic organizational } \\
\text { culture (DOC) }\end{array}$ & $\begin{array}{c}\text { [24],[41],[28],[23], } \\
{[18],[12],[13]}\end{array}$ \\
\hline 9. & $\begin{array}{l}\text { Increased opportunity for } \\
\text { innovation and value } \\
\text { engineering (IOIVE) }\end{array}$ & {$[24],[41]$} \\
\hline 10. & $\begin{array}{l}\text { Reduced exposure to } \\
\text { litigation (REL) }\end{array}$ & {$[24],[41]$} \\
\hline 11. & $\begin{array}{c}\text { Improved project outcomes } \\
\text { in terms of cost , time and } \\
\text { quality (IPO) }\end{array}$ & [24], [41] \\
\hline 12. & $\begin{array}{l}\text { Making effort to build long } \\
\text { term profitability (LTP) }\end{array}$ & [24], [41] \\
\hline 13. & $\begin{array}{l}\text { Lower administrative and } \\
\text { legal costs (LALC) }\end{array}$ & [24], [41] \\
\hline 14. & $\begin{array}{l}\text { Achieving better buildbility } \\
\text { (ABB) }\end{array}$ & [24], [41] \\
\hline 15. & $\begin{array}{c}\text { Long term quality } \\
\text { perspectives (LTQP) and } \\
\text { thereby developing win-win } \\
\text { solution }\end{array}$ & [24], [41] \\
\hline 16. & Technical expertise (TE) & [24], [41] \\
\hline
\end{tabular}

\section{INTERPRETIVE STRUCTURAL MODELLING METHODOLOGY}

Suggested by [43], the technique has been used widely to develop a map of the relationships between the many elements in the form of a hierarchy graph. Group's judgement decides whether and how the items are related. Steps in applying an ISM methodology involves identification of relevant elements and then establishing contextual relationship amongst them taking elements pairwise. Further to this a SSIM i.e. the structural self- interaction matrix has been developed followed by initial and then final reachability matrix [Warfield (1994)] . A dependence and driving element diagram and a diagraph can be obtained afterwards to see the hierarchical relationship amongst the relevant elements .

\section{DEVELOPMENT OF ISM MODEL}

In this section, ISM model is developed for studying the interrelationships amongst various success factors for partnering in construction projects supply chains. Sixteen important success factors viz . Effective communication / clear lines of communication (CLC); Effective co-ordination (EC) ; better resource sharing (BRS); Dedicated team culture (DTC); Long term relationships (LTR) ; Mutual objectives/ goals and mutual trust (MO/MT) ; Management support (MS) and financial security ; Dynamic organizational culture (DOC) ; Increased opportunity for innovation and value engineering (IOIVE) ; Reduced exposure to litigation (REL) ; Improved project outcomes in terms of cost , time and quality (IPO) ; Making effort to build long term profitability (LTP) ; Lower administrative and legal costs (LALC) ; Achieving 
better buildability (ABB) ; Long term quality perspectives (LTQP) and thereby developing win-win solution ; Technical expertise (TE) have been identified for studying further through ISM methodology .

\subsection{Construction of Structural Self - Interaction Matrix (SSIM)}

This matrix gives the pair-wise relationship between two variables i.e. $i$ and $j$ based on VAXO. SSIM has been presented below in Fig 1.

\section{Explanation:}

Dedicated team culture will lead to better co-ordination amongst team members and better resource sharing. Better resource sharing will lead to long term relationship and vice versa . Mutual objectives will lead to better resource sharing, better co-ordination. Dedicated team culture will help in achieving mutual objectives and establishing mutual trust. Long term relationship will lead to improved project outcomes. Management support can be obtained with mutual trust, objectives and dedicated team culture. Dynamic organizational culture will lead to improved project outcomes Increased opportunity for innovation may lead to long term profitability . Dynamic organizational culture may demand technical expertise from its employees . Long term quality perspectives may demand technical expertise and vice versa.

\subsection{Construction of Initial Reachability} Matrix and final reachability matrix

The SSIM has been converted in to a binary matrix called the initial reachability matrix shown in fig. 2 by substituting $\mathrm{V}, \mathrm{A}$, $\mathrm{X}, \mathrm{O}$ by 1 or 0 as per the case. After incorporating the transitivity, the final reachability matrix is shown below in the Fig 3.

Fig 1: SSIM matrix for pair wise relationship amongst barriers

\begin{tabular}{|c|c|c|c|c|c|c|c|c|c|c|c|c|c|c|c|c|c|}
\hline S.no. & $\begin{array}{l}\text { Success } \\
\text { factors }\end{array}$ & $\mathbf{1}$ & $\mathbf{2}$ & $\mathbf{3}$ & $\mathbf{4}$ & $\mathbf{5}$ & $\mathbf{6}$ & $\mathbf{7}$ & $\mathbf{8}$ & $\mathbf{9}$ & $\mathbf{1 0}$ & $\mathbf{1 1}$ & $\mathbf{1 2}$ & $\mathbf{1 3}$ & $\mathbf{1 4}$ & $\mathbf{1 5}$ & $\mathbf{1 6}$ \\
\hline & & CLC & EC & BRS & DTC & LTR & MO & MS & DOC & IOIVE & REL & IPO & LTP & LALC & ABB & LTQP & TE \\
\hline 1 & CLC & & V & V & V & V & V & V & V & V & V & V & V & V & V & V & O \\
\hline 2 & EC & & & V & A & V & A & V & A & V & V & V & V & V & V & V & A \\
\hline 3 & BRS & & & A & X & A & A & A & V & V & V & V & V & V & V & A \\
\hline 4 & DTC & & & & V & V & V & X & V & V & V & V & V & V & V & O \\
\hline 5 & LTR & & & & & V & V & X & V & V & V & V & V & V & V & O \\
\hline 6 & MO/MT & & & & & & & V & X & V & V & V & V & V & V & V & O \\
\hline 7 & MS & & & & & & & A & V & V & V & V & V & V & V & A \\
\hline 8 & DOC & & & & & & & & V & V & V & V & V & V & V & V \\
\hline 9 & IOIVE & & & & & & & & & & A & A & V & V & V & V & A \\
\hline 10 & REL & & & & & & & & & & & V & V & V & V & V & O \\
\hline 11 & IPO & & & & & & & & & & & & V & A & A & A & A \\
\hline 12 & LTP & & & & & & & & & & & & V & A & A & A \\
\hline 13 & LALC & & & & & & & & & & & & & V & O & A \\
\hline 14 & ABB & & & & & & & & & & & & & A & A \\
\hline 15 & LTQP & & & & & & & & & & & & & & & X \\
\hline 16 & TE & & & & & & & & & & & & & & & \\
\hline
\end{tabular}


Fig 2: Initial reachability matrix

\begin{tabular}{|c|c|c|c|c|c|c|c|c|c|c|c|c|c|c|c|c|c|}
\hline S.no. & $\begin{array}{l}\text { Success } \\
\text { factors }\end{array}$ & 1 & 2 & 3 & 4 & 5 & 6 & 7 & 8 & 9 & 10 & 11 & 12 & 13 & 14 & 15 & 16 \\
\hline & & CLC & EC & BRS & DTC & LTR & MO & MS & DOC & IOIVE & REL & IPO & LTP & LALC & $\mathrm{ABB}$ & LTQP & $\mathrm{TE}$ \\
\hline 1 & CLC & 1 & 1 & 1 & 1 & 1 & 1 & 1 & 1 & 1 & 1 & 1 & 1 & 1 & 1 & 1 & 0 \\
\hline 2 & EC & 0 & 1 & 1 & 0 & 1 & 0 & 1 & 0 & 1 & 1 & 1 & 1 & 1 & 1 & 1 & 0 \\
\hline 3 & BRS & 0 & 0 & 1 & 0 & 1 & 0 & 0 & 0 & 1 & 1 & 1 & 1 & 1 & 1 & 1 & 0 \\
\hline 4 & DTC & 0 & 1 & 1 & 1 & 1 & 1 & 1 & 1 & 1 & 1 & 1 & 1 & 1 & 1 & 1 & 0 \\
\hline 5 & LTR & 0 & 0 & 1 & 0 & 1 & 1 & 1 & 1 & 1 & 1 & 1 & 1 & 1 & 1 & 1 & 0 \\
\hline 6 & $\mathrm{MO} / \mathrm{MT}$ & 0 & 1 & 1 & 0 & 0 & 1 & 1 & 1 & 1 & 1 & 1 & 1 & 1 & 1 & 1 & 0 \\
\hline 7 & MS & 0 & 0 & 1 & 0 & 0 & 0 & 1 & 0 & 1 & 1 & 1 & 1 & 1 & 1 & 1 & 0 \\
\hline 8 & DOC & 0 & 1 & 1 & 1 & 1 & 1 & 1 & 1 & 1 & 1 & 1 & 1 & 1 & 1 & 1 & 1 \\
\hline 9 & IOIVE & 0 & 0 & 0 & 0 & 0 & 0 & 0 & 0 & 1 & 0 & 0 & 1 & 1 & 1 & 1 & 0 \\
\hline 10 & REL & 0 & 0 & 0 & 0 & 0 & 0 & 0 & 0 & 1 & 1 & 1 & 1 & 1 & 1 & 1 & 0 \\
\hline 11 & IPO & 0 & 0 & 0 & 0 & 0 & 0 & 0 & 0 & 1 & 0 & 1 & 1 & 0 & 0 & 0 & 0 \\
\hline 12 & LTP & 0 & 0 & 0 & 0 & 0 & 0 & 0 & 0 & 0 & 0 & 0 & 1 & 1 & 0 & 0 & 0 \\
\hline 13 & LALC & 0 & 0 & 0 & 0 & 0 & 0 & 0 & 0 & 0 & 0 & 1 & 0 & 1 & 1 & 0 & 0 \\
\hline 14 & $\mathrm{ABB}$ & 0 & 0 & 0 & 0 & 0 & 0 & 0 & 0 & 0 & 0 & 1 & 1 & 0 & 1 & 0 & 0 \\
\hline 15 & LTQP & 0 & 0 & 0 & 0 & 0 & 0 & 0 & 0 & 0 & 0 & 1 & 1 & 0 & 1 & 1 & 1 \\
\hline 16 & TE & 0 & 1 & 1 & 0 & 0 & 0 & 1 & 0 & 1 & 0 & 1 & 1 & 1 & 1 & 1 & 1 \\
\hline
\end{tabular}

Fig 3 : Final reachability matrix

\begin{tabular}{|c|c|c|c|c|c|c|c|c|c|c|c|c|c|c|c|c|c|c|}
\hline S.no. & $\begin{array}{l}\text { Success } \\
\text { factors }\end{array}$ & 1 & 2 & 3 & 4 & 5 & 6 & 7 & 8 & 9 & 10 & 11 & 12 & 13 & 14 & 15 & 16 & D.P \\
\hline & & CLC & $\mathrm{EC}$ & BRS & DTC & LTR & MO & MS & DOC & IOIVE & REL & IPO & LTP & LALC & ABB & LTQP & TE & \\
\hline 1 & CLC & 1 & 1 & 1 & 1 & 1 & 1 & 1 & 1 & 1 & 1 & 1 & 1 & 1 & 1 & 1 & 1 & 16 \\
\hline 2 & $\mathrm{EC}$ & 0 & 1 & 1 & 0 & 1 & 0 & 1 & 0 & 1 & 1 & 1 & 1 & 1 & 1 & 1 & 1 & 12 \\
\hline 3 & BRS & 0 & 0 & 1 & 0 & 1 & 0 & 0 & 0 & 1 & 1 & 1 & 1 & 1 & 1 & 1 & 1 & 10 \\
\hline 4 & DTC & 0 & 1 & 1 & 1 & 1 & 1 & 1 & 1 & 1 & 1 & 1 & 1 & 1 & 1 & 1 & 1 & 15 \\
\hline 5 & LTR & 0 & 0 & 1 & 0 & 1 & 1 & 1 & 1 & 1 & 1 & 1 & 1 & 1 & 1 & 1 & 1 & 13 \\
\hline 6 & $\mathrm{MO} / \mathrm{MT}$ & 0 & 1 & 1 & 0 & 1 & 1 & 1 & 1 & 1 & 1 & 1 & 1 & 1 & 1 & 1 & 1 & 14 \\
\hline 7 & MS & 0 & 0 & 1 & 0 & 0 & 0 & 1 & 0 & 1 & 1 & 1 & 1 & 1 & 1 & 1 & 1 & 10 \\
\hline 8 & $\overline{\mathrm{DOC}}$ & 0 & 1 & 1 & 1 & 1 & 1 & 1 & 1 & 1 & 1 & 1 & 1 & 1 & 1 & 1 & 1 & 15 \\
\hline 9 & IOIVE & 0 & 0 & 0 & 0 & 0 & 0 & 0 & 0 & 1 & 0 & 1 & 1 & 1 & 1 & 1 & 1 & 7 \\
\hline 10 & REL & 0 & 0 & 0 & 0 & 0 & 0 & 0 & 0 & 1 & 1 & 1 & 1 & 1 & 1 & 1 & 1 & 8 \\
\hline 11 & IPO & 0 & 0 & 0 & 0 & 0 & 0 & 0 & 0 & 1 & 0 & 1 & 1 & 1 & 1 & 1 & 0 & 6 \\
\hline 12 & LTP & 0 & 0 & 0 & 0 & 0 & 0 & 0 & 0 & 0 & 0 & 0 & 1 & 1 & 1 & 0 & 0 & 3 \\
\hline 13 & LALC & 0 & 0 & 0 & 0 & 0 & 0 & 0 & 0 & 1 & 0 & 1 & 1 & 1 & 1 & 0 & 0 & 5 \\
\hline 14 & $\overline{\mathrm{ABB}}$ & 0 & 0 & 0 & 0 & 0 & 0 & 0 & 0 & 1 & 0 & 1 & 1 & 1 & 1 & 0 & 0 & 5 \\
\hline 15 & LTQP & 0 & 0 & 0 & 0 & 0 & 0 & 0 & 0 & 1 & 0 & 1 & 1 & 1 & 1 & 1 & 1 & 7 \\
\hline \multirow[t]{2}{*}{16} & $\mathrm{TE}$ & 0 & 1 & 1 & 0 & 0 & 0 & 1 & 0 & 1 & 1 & 1 & 1 & 1 & 1 & 1 & 1 & 11 \\
\hline & De. $P$ & 1 & 6 & 9 & 4 & 7 & 5 & 8 & 6 & 15 & 10 & 15 & 16 & 16 & 16 & 13 & 12 & \\
\hline
\end{tabular}




\subsection{Level Partition}

Table 2 : Iteration I

\begin{tabular}{|c|c|c|c|c|}
\hline S.No. & $\begin{array}{c}\text { Reachabilit } \\
\text { y set }\end{array}$ & $\begin{array}{c}\text { Antecedent } \\
\text { set }\end{array}$ & $\begin{array}{c}\text { Intersectio } \\
\text { n set }\end{array}$ & $\begin{array}{c}\text { Iteratio } \\
\mathbf{n} / \\
\text { Levels }\end{array}$ \\
\hline 1. & $12,13,14$ & $\begin{array}{c}1,2,3,4,5,6,7,8 \\
9,10,11,12,13,1 \\
4,15,16\end{array}$ & $12,13,14$ & \\
\hline 2. & $\begin{array}{c}9, \\
11,12,13,14\end{array}$ & $\begin{array}{c}1,2,3,4,5,6,7,8 \\
9,10,11,13,14,1 \\
5,16\end{array}$ & $9,11,13.14$ & \\
\hline 3. & $\begin{array}{c}9,11,12,13,1 \\
4,15\end{array}$ & $\begin{array}{c}1,2,3,4,5,6,7,8 \\
9,10,11,15,16\end{array}$ & $9,11,15$ & \\
\hline 4. & $\begin{array}{c}9,11,12,13,1 \\
4,15,16\end{array}$ & $\begin{array}{c}1,2,3,4,5,6,7,8 \\
9,10,15,16\end{array}$ & $9,15,16$ & \\
\hline 5. & $\begin{array}{c}9,10,11,12,1 \\
3,14,15,16\end{array}$ & $\begin{array}{c}1,2,3,4,5,6,7,8 \\
10,15,16\end{array}$ & $10,15,16$ & \\
\hline 6. & $\begin{array}{l}3,9,10,11,12 \\
, 13,14,15,16\end{array}$ & $\begin{array}{c}1,2,3,4,5,6,7,8 \\
16\end{array}$ & 3,16 & \\
\hline 7. & $\begin{array}{c}3,7,9,10,11 \\
12,13,14,15 \\
16\end{array}$ & $1,2,4,5,6,7,8,16$ & 7,16 & \\
\hline 8. & $\begin{array}{c}3,5,7,9,10,1 \\
1,12,13,14,1 \\
5,16\end{array}$ & $1,2,4,5,8,16$ & 5,16 & I \\
\hline 9. & $\begin{array}{c}3,5,7,8,9,10 \\
11,12,13,14 \\
15,16\end{array}$ & $1,2,4,5,7,8,16$ & $5,7,8,16$ & \\
\hline 10. & $\begin{array}{c}2,3,5,7,8,9,1 \\
0,11,12,13,1 \\
4,15,16\end{array}$ & $1,2,4,6,7,8,16$ & $2,7,8,16$ & \\
\hline 11. & $\begin{array}{c}2,3,5,6,7,8,9 \\
10,11,12,13 \\
, 14,15,16\end{array}$ & $1,4,6,7,8,16$ & $6,7,8,16$ & \\
\hline 12. & $\begin{array}{c}2,3,4,5,6,7,8 \\
, 9,10,11,12, \\
13,14,15,16\end{array}$ & $1,4,8$ & 4,8 & \\
\hline 13. & $\begin{array}{c}1,2,3,4,5,6,7 \\
, 8,9,10,11,1 \\
2,13,14,15,1 \\
6\end{array}$ & 1 & 1 & \\
\hline
\end{tabular}

From the final reachability matrix, reachability and final antecedent set for each factor are found. The element for which the reachability and intersection sets are same are the top-level element in the ISM hierarchy. After the identification of top level element, it is separated out from the other elements and the process continues for next level of elements. Reachability set, antecedent set, intersection set along with different level for elements have been shown below in table II to table XI.
Table 3 : Iteration II

\begin{tabular}{|c|c|c|c|c|}
\hline S.No. & $\begin{array}{l}\text { Reachabili } \\
\text { ty set }\end{array}$ & Antecedent set & $\begin{array}{c}\text { Intersection } \\
\text { set }\end{array}$ & $\begin{array}{l}\text { Itera } \\
\text { tion }\end{array}$ \\
\hline 2. & 9,11 & $\begin{array}{c}1,2,3,4,5,6,7,8 \\
9,10,11,15,16\end{array}$ & 9,11 & \multirow{12}{*}{ II } \\
\hline 3. & $9,11,15$ & $\begin{array}{c}1,2,3,4,5,6,7,8 \\
9,10,11,15,16\end{array}$ & $9,11,15$ & \\
\hline 4. & $9,11,15,16$ & $\begin{array}{c}1,2,3,4,5,6,7,8 \\
9,10,15,16\end{array}$ & $9,15,16$ & \\
\hline 5. & $\begin{array}{c}9,10,11,15 \\
16\end{array}$ & $\begin{array}{c}1,2,3,4,5,6,7,8 \\
10,15,16\end{array}$ & $10,15,16$ & \\
\hline 6. & $\begin{array}{c}3,9,10,11,1 \\
5,16\end{array}$ & $\begin{array}{c}1,2,3,4,5,6,7,8 \\
16\end{array}$ & 3,16 & \\
\hline 7. & $\begin{array}{c}3,7,9,10,11, \\
15,16\end{array}$ & $1,2,4,5,6,7,8,16$ & 7,16 & \\
\hline 8. & $\begin{array}{c}3,5,7,9,10,1 \\
1,15,16\end{array}$ & $1,2,4,5,8,16$ & 5,16 & \\
\hline 9. & $\begin{array}{c}3,5,7,8,9,10 \\
, 11,15,16\end{array}$ & $1,2,4,5,7,8,16$ & $5,7,8,16$ & \\
\hline 10. & $\begin{array}{l}2,3,5,7,8,9, \\
10,11,15,16\end{array}$ & $1,2,4,6,7,8,16$ & $2,7,8,16$ & \\
\hline 11. & $\begin{array}{c}2,3,5,6,7,8 \\
9,10,11,15 \\
16\end{array}$ & $1,4,6,7,8,16$ & $6,7,8,16$ & \\
\hline 12. & $\begin{array}{c}2,3,4,5,6,7 \\
8,9,10,11,1 \\
5,16\end{array}$ & $1,4,8$ & 4,8 & \\
\hline 13. & $\begin{array}{c}1,2,3,4,5,6 \\
7,8,9,10,11 \\
15,16\end{array}$ & 1 & 1 & \\
\hline
\end{tabular}

Table 4 : Iteration III

\begin{tabular}{|c|c|c|c|c|}
\hline $\begin{array}{l}\text { Sr. } \\
\text { No. }\end{array}$ & $\begin{array}{c}\text { Reachability } \\
\text { set }\end{array}$ & $\begin{array}{c}\text { Antecedent } \\
\text { set }\end{array}$ & $\begin{array}{c}\text { Intersection } \\
\text { set }\end{array}$ & $\begin{array}{l}\text { Itera } \\
\text { tion }\end{array}$ \\
\hline 4 & 16 & $\begin{array}{c}1,2,3,4,5,6,7 \\
8,10,16\end{array}$ & 16 & \multirow{9}{*}{ III } \\
\hline 5 & 10,16 & $\begin{array}{c}1,2,3,4,5,6,7 \\
8,10,16\end{array}$ & 10,16 & \\
\hline 6 & $3,10,16$ & $\begin{array}{c}1,2,3,4,5,6,7, \\
8,16\end{array}$ & 3,16 & \\
\hline 7 & $3,7,10,16$ & $\begin{array}{c}1,2,4,5,6,7,8 \\
16\end{array}$ & 7,16 & \\
\hline 8 & $3,5,7,10,16$ & $1,2,4,5,8,16$ & 5,16 & \\
\hline 9 & $3,5,7,8,10,16$ & $\begin{array}{c}1,2,4,5,7,8,1 \\
6\end{array}$ & $5,7,8,16$ & \\
\hline 10 & $2,3,5,7,8,10,16$ & $\begin{array}{c}1,2,4,6,7,8,1 \\
6\end{array}$ & $2,7,8,16$ & \\
\hline 11 & $\begin{array}{c}2,3,5,6,7,8,10 \\
16\end{array}$ & $1,4,6,7,8,16$ & $6,7,8,16$ & \\
\hline 12 & $\begin{array}{c}2,3,4,5,6,7,8,1 \\
0,16\end{array}$ & $1,4,8$ & 4,8 & \\
\hline
\end{tabular}




\begin{tabular}{|c|c|c|c|c|}
\hline 13 & $\begin{array}{c}1,2,3,4,5,6,7,8, \\
10,16\end{array}$ & 1 & 1 & \\
\hline 4 & $\mathbf{1 6}$ & $\begin{array}{c}1,2,3,4,5,6,7, \\
8,10,16\end{array}$ & $\mathbf{1 6}$ & \\
\hline
\end{tabular}

Table 6 : Iteration IV

\begin{tabular}{|c|c|c|c|c|}
\hline S.No. & $\begin{array}{c}\text { Reachabilit } \\
\text { y set }\end{array}$ & Antecedent set & $\begin{array}{c}\text { Intersecti } \\
\text { on set }\end{array}$ & $\begin{array}{c}\text { Iterat } \\
\text { ion/ } \\
\text { Level } \\
\text { s }\end{array}$ \\
\hline 5 & 10 & $\begin{array}{c}1,2,3,4,5,6,7,8 \\
10\end{array}$ & 10 & \multirow{9}{*}{ IV } \\
\hline 6 & 3,10 & $1,2,3,4,5,6,7,8$ & 3 & \\
\hline 7 & $3,7,10$ & $1,2,4,5,6,7,8$ & 7 & \\
\hline 8 & $3,5,7,10$ & $1,2,4,5,8$ & 5 & \\
\hline 9 & $3,5,7,8,10$ & $1,2,4,5,7,8$ & $5,7,8$ & \\
\hline 10 & $2,3,5,7,8,10$ & $1,2,4,6,7,8$ & $2,7,8$ & \\
\hline 11 & $\begin{array}{c}2,3,5,6,7,8,1 \\
0\end{array}$ & $1,4,6,7,8$ & $6,7,8$ & \\
\hline 12 & $\begin{array}{c}2,3,4,5,6,7,8 \\
, 10\end{array}$ & $1,4,8$ & 4,8 & \\
\hline 13 & $\begin{array}{c}1,2,3,4,5,6,7 \\
, 8,10\end{array}$ & 1 & 1 & \\
\hline
\end{tabular}

Table 7 : Iteration V

\begin{tabular}{|c|c|c|c|c|}
\hline $\begin{array}{l}\text { Sr. } \\
\text { No. }\end{array}$ & $\begin{array}{c}\text { Reachability } \\
\text { set }\end{array}$ & $\begin{array}{c}\text { Antecedent } \\
\text { set }\end{array}$ & $\begin{array}{c}\text { Intersection } \\
\text { set }\end{array}$ & $\begin{array}{l}\text { Itera } \\
\text { tion }\end{array}$ \\
\hline 6 & 3 & $\begin{array}{c}1,2,3,4,5,6,7 \\
8\end{array}$ & 3 & \multirow{8}{*}{$\mathbf{V}$} \\
\hline 7 & 3,7 & $1,2,4,5,6,7,8$ & 7 & \\
\hline 8 & $3,5,7$ & $1,2,4,5,8$ & 5 & \\
\hline 9 & $3,5,7,8$ & $1,2,4,5,7,8$ & $5,7,8$ & \\
\hline 10 & $2,3,5,7,8$ & $1,2,4,6,7,8$ & $2,7,8$ & \\
\hline 11 & $2,3,5,6,7,8$ & $1,4,6,7,8$ & $6,7,8$ & \\
\hline 12 & $2,3,4,5,6,7,8$ & $1,4,8$ & 4,8 & \\
\hline 13 & $\begin{array}{c}1,2,3,4,5,6,7,8 \\
12,13,14\end{array}$ & 1 & 1 & \\
\hline
\end{tabular}

Table 8 : Iteration VI

\begin{tabular}{|c|c|c|c|}
\hline $\begin{array}{l}\text { Sr. } \\
\text { No. }\end{array}$ & $\begin{array}{c}\text { Reachability } \\
\text { set }\end{array}$ & Antecedent set & $\begin{array}{c}\text { Intersection } \\
\text { set }\end{array}$ \\
\hline 7 & 7 & $1,2,4,5,6,7,8$ & 7 \\
\hline 8 & 5,7 & $1,2,4,5,8$ & 5 \\
\hline 9 & $5,7,8$ & $1,2,4,5,7,8$ & $5,7,8$ \\
\hline 10 & $2,5,7,8$ & $1,2,4,6,7,8$ & $2,7,8$ \\
\hline 11 & $2,5,6,7,8$ & $1,4,6,7,8$ & $6,7,8$ \\
\hline 12 & $2,4,5,6,7,8$ & $1,4,8$ & 4,8 \\
\hline 13 & $1,2,4,5,6,7,8$ & 1 & 1 \\
\hline 7 & 7 & $1,2,4,5,6,7,8$ & 7 \\
\hline
\end{tabular}

Table 9 : Iteration VII

\begin{tabular}{|c|c|c|c|c|}
\hline $\begin{array}{l}\text { Sr. } \\
\text { No. }\end{array}$ & $\begin{array}{c}\text { Reachability } \\
\text { set }\end{array}$ & $\begin{array}{c}\text { Antecedent } \\
\text { set }\end{array}$ & $\begin{array}{c}\text { Intersection } \\
\text { set }\end{array}$ & $\begin{array}{c}\text { Itera } \\
\text { tion }\end{array}$ \\
\hline 8 & 5 & $1,2,4,5,8$ & 5 & \multirow{7}{*}{ VII } \\
\hline 9 & 5,8 & $1,2,4,5,8$ & 5,8 & \\
\hline 10 & $2,5,8$ & $1,2,4,6,8$ & 2,8 & \\
\hline 11 & $2,5,6,8$ & $1,4,6,8$ & 6,8 & \\
\hline 2 & $2,4,5,6,8$ & $1,4,8$ & 4,8 & \\
\hline \multirow[t]{2}{*}{13} & $1,2,4,5,6,8$ & 1 & 1 & \\
\hline & 5 & $1,2,4,5,8$ & 5 & \\
\hline
\end{tabular}

Table 10 : Iteration VIII

\begin{tabular}{|c|c|c|c|c|}
\hline $\begin{array}{c}\text { Sr. } \\
\text { No. }\end{array}$ & $\begin{array}{c}\text { Reachability } \\
\text { set }\end{array}$ & $\begin{array}{c}\text { Antecedent } \\
\text { set }\end{array}$ & $\begin{array}{c}\text { Intersection } \\
\text { set }\end{array}$ & $\begin{array}{c}\text { Itera } \\
\text { tion }\end{array}$ \\
\hline 10 & $\mathbf{2}$ & $1,2,4,6$ & 2 & \\
\hline 11 & 2,6 & $1,4,6$ & 6 & \multirow{2}{*}{ VIII } \\
\hline 12 & $2,4,6$ & 1,4 & 4 & \\
\hline 13 & $1,2,4,6$ & 1 & 1 & \\
\hline
\end{tabular}

Table 11 : Iteration IX

\begin{tabular}{|c|c|c|c|c|}
\hline $\begin{array}{c}\text { Sr. } \\
\text { No. }\end{array}$ & $\begin{array}{c}\text { Reachability } \\
\text { set }\end{array}$ & $\begin{array}{c}\text { Antecedent } \\
\text { set }\end{array}$ & $\begin{array}{c}\text { Intersection } \\
\text { set }\end{array}$ & $\begin{array}{c}\text { Itera } \\
\text { tion }\end{array}$ \\
\hline 11 & $\mathbf{6}$ & $1,4,6$ & 6 & \\
\hline 12 & 4,6 & 1,4 & 4 & \multirow{2}{*}{ IX } \\
\hline 13 & $1,4,6$ & 1 & 1 & \\
\hline
\end{tabular}


Table 12 : Iteration $\mathrm{X}$

\begin{tabular}{|c|c|c|c|c|}
\hline $\begin{array}{c}\text { Sr. } \\
\text { No. }\end{array}$ & $\begin{array}{c}\text { Reachability } \\
\text { set }\end{array}$ & $\begin{array}{c}\text { Antecedent } \\
\text { set }\end{array}$ & $\begin{array}{c}\text { Intersection } \\
\text { set }\end{array}$ & $\begin{array}{c}\text { Itera } \\
\text { tion }\end{array}$ \\
\hline 12 & $\mathbf{4}$ & 1,4 & 4 & \\
\hline 13 & 1,4 & 1 & 1 & \\
& & & & $\mathbf{X}$ \\
\hline
\end{tabular}

Table 12 : Iteration XI

\begin{tabular}{|c|c|c|c|c|}
\hline $\begin{array}{c}\text { Sr. } \\
\text { No. }\end{array}$ & $\begin{array}{c}\text { Reachability } \\
\text { set }\end{array}$ & $\begin{array}{c}\text { Antecedent } \\
\text { set }\end{array}$ & $\begin{array}{c}\text { Intersection } \\
\text { set }\end{array}$ & $\begin{array}{c}\text { Itera } \\
\text { tion }\end{array}$ \\
\hline 13 & 1 & 1 & 1 & XI \\
\hline
\end{tabular}

\subsection{Classification of factors}

The critical success factors described earlier are classified in to four clusters viz. autonomous factor, dependent factors, linkage factors and independent factors (mentioned in Table XIII below). As it can be seen that CLC is an autonomous criteria. Criteria DTC, DOC, LTR, EC, MO , MS are drivers . Criteria such as REL, LTQP , IOIVE, LALC, ABB , LTP are dependent criteria. Criteria TE and BRS are linkage criteria.

Fig. 4 below shows the driving power and dominance diagram

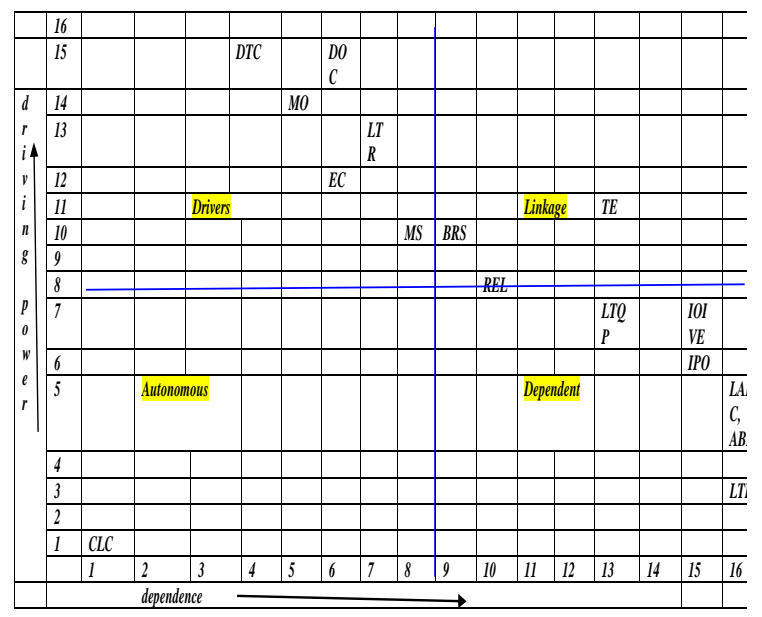

Fig . 4: Driving power and dependence diagram

\subsection{ISM model}

An ISM model is developed ( as shown in fig. 5 below ) after arranging the elements as per their interaction or dependence relationships.

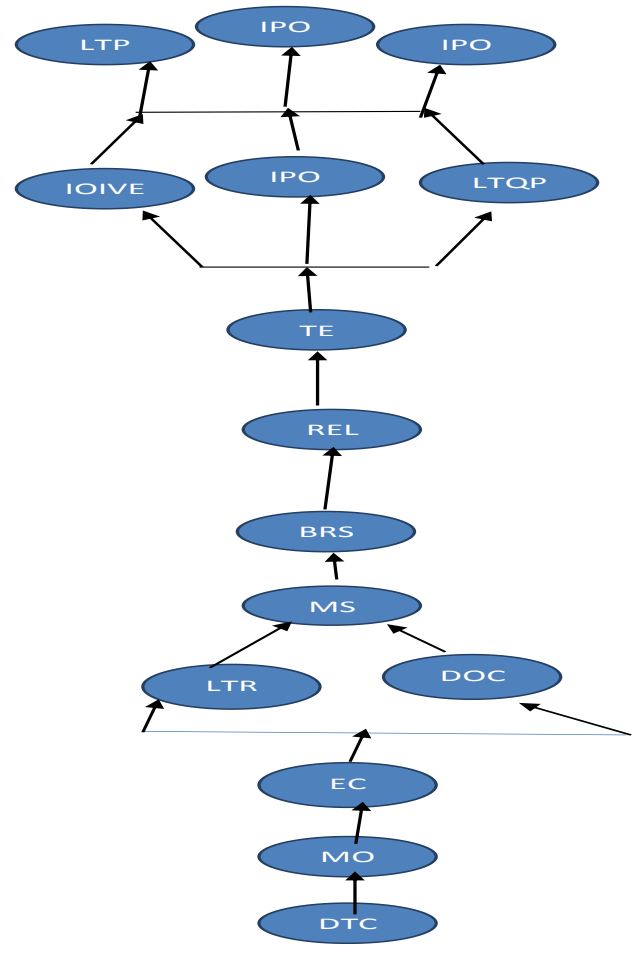

Fig 5: ISM diagraph

\section{CONCLUSIONS}

The following research focuses on exploring various success factors for successful partnering in construction industry in developing countries like India. It further tries to explore the interrelationship amongst the factors using ISM methodology

\section{ACKNOWLEDGEMENT}

Authors are thankful to Prof. S.P Singh, Department of Management Studies for imparting knowledge on ISM methodology which helped authors substantially while writing this piece of work.

\section{REFERENCES}

[1] Vrijhoef, R. and Koskela, L. 2000. The four roles of supply chain management in construction. European Journal of Purchasing \& Supply Management, 6(3-4), 169-178.

[2] Dubois, A. and Gadde, L.-E. 2002. The construction industry as a loosely coupled system: implications for productivity and innovation, Construction Management \& Economics, 20 (7), 621-631.

[3] Gadde, L.E. \& Dubois, A. 2010. Partnering in the construction industry-Problems and opportunities". Journal of Purchasing and Supply Management, 16, 254263.

[4] Bakker, René M. (2010). "Taking Stock of Temporary Organizational Forms: A Systematic Review and Research Agenda". International Journal of Management Reviews, 12, 466-486.

[5] Ekeskär, A. 2016.. Exploring Third-Party Logistics and Partnering in Construction - A Supply Chain Management Perspective , Linköping Studies in Science and Technology. Licentiate Thesis No. 1753

[6] Agapiou, A., Flanagan, R., Norman, G. and Notman, D. 
1998b. The changing role of builders merchants in the construction supply chain . Construction Management and Economics, 16, 351-361.

[7] Vidalakis, C. \& Sommerville, J. 2013. "Transportation responsiveness and efficiency within the building supply chain". Building Research \& Information, 41, 469-481.

[8] Miller, C. J. M., Packham, G. A. and Thomas, B. C. 2002. Harmonization between Main Contractors and Subcontractors: A Prerequisite for Lean Construction?, Journal of Construction Research, 3(1), 67-82.

[9] Winch, G. M. 2001. Governing the project process: a conceptual framework. Construction Management and Economics, 19, 799-808.

[10] CII, Construction Industry Institute 1991. In Search of Partnering Excellence. CII Special Publication, Austin, Texas: Construction Industry Institute.

[11] CII, Construction Industry Institute (2015). CII Best Practices [Online]. Available: http://bit.ly/1U8FD01 [Accessed: 7th of March 2016].

[12] Kadefors, A. 1995. "Institutions in building projects: Implications for flexibility and change". Scandinavian Journal of Management, 11, 395-408.

[13] Kadefors, Anna 2011. "Samarbete i byggandet - igår, idag och i framtiden". In: Landin, A. \& Lind, H. (eds.) Hur står det egentligen till med den Svenska byggsektorn? Perspektiv från forskarvärlden. pp. Lund: Lunds universitet

[14] Saad, M., Jones, M. \& James, P. 2002. A review of the progress towards the adoption of supply chain management (SCM) relationships in construction". European Journal of Purchasing \& Supply Management, 8, 173-183.

[15] Li, H., Cheng, E.W. L. \& Love, P.E. D. (2000). Partnering research in construction. Engineering Construction \& Architectural Management (WileyBlackwell), 7, 76-92.

[16] Crespin-Mazet, F., Ingemansson H.M. \& Linné, Å. 2015. Antecedents of project partnering in the construction industry - The impact of relationship history. Industrial Marketing Management, 50, 4-15.

[17] Eriksson, P.E. 2010. Partnering: what is it, when should it be used, and how should it be implemented? Construction Management and Economics, 28, 905-917.

[18] Manchester Business School (2009). Study on voluntary arrangements for collaborative working in the field of construction services, Brussels: European Commission DG Enterprise and Industry.

[19] Bresnen, M. \& Marshall, N. 2000a. Partnering in construction: a critical review of issues, problems and dilemmas. Construction Management and Economics, $18,229-237$.

[20] Bresnen, M. \& Marshall, N. (2000b). Building partnerships: case studies of client-contractor collaboration in the UK construction industry. Construction Management \& Economics, 18, 819-832.

[21] Egan, J. 1998. Rethinking Construction: Report of the Construction Task Force, HMSO, London.

[22] Jacobsson, M. \& Roth, P. 2014. Towards a shift in mindset: partnering projects as engagement platforms Construction Management \& Economics, 32, 419-432.

[23] Bygballe, L.E., Jahre, M. \& Swärd, A. 2010. Partnering relationships in construction: A literature review. Journal of Purchasing and Supply Management, 16, 239-253.

[24] Awodele, O.A. and Ogunsemi, D.R. 2007. An Assessment of Success Factors and Benefits of Project Partnering in Nigerian Construction Industrywww.academia.edu/.../An Assessment_of_Succe ss_Factors_and_Benefits_of_Project_Partnering_in_Nige rian_Construction_Industry

[25] Chadwick, T. and Rajagopal, S. (1995). Strategic supplying management. London: ButterworthHeinenmann. 92-117.

[26] Cheng, E. W. L. \& Li, H. 2002. Construction Partnering Process and Associated Critical Success Factors: Quantitative Investigation. Journal of Management in Engineering, 18, 194

[27] Black, C., Akintoye, A., Fitzgerald, E. 2000. An Analysis of Success Factors and Benefits of Partnering in Construction, International Journal of Project Management, 18(6), 423-34.

[28] Chan, A.P.C., Chan, D.W.M., Chiang, Y.H., Tang, B.S., Chan, E.H.W., HO, K.S.K. 2004. Exploring Critical Success Factors for Partnering in Construction Projects, Journal of Construction Engineering and Management , 130(2) 188-98.

[29] Nystrom, J. 2005. The Definition of Partnering as a Wittgenstein Family Resemblance Concept, Construction Management and Economics 23(5) 473-81.

[30] Hong, Y., Chan, D., Chan, A., Yeung, J. (2012) Critical Analysis of Partnering Research Trend in Construction Journals, Journal of Management in Engineering, 28(2) 82-95.

[31] Karrbom , G. T. \& Hallin, A. 2014. Rethinking dichotomization: A critical perspective on the use of "hard" and "soft" in project management research. International Journal of Project Management, 32, 568577.

[32] Dainty, A. R. J., Briscoe, G. H. \& Millett, S. J. 2001a. Subcontractor perspectives on supply chain alliances. Construction Management and Economics, 19, 841-848.

[33] Wolstenholme, A. 2009. Never Waste a Good Crisis A Review of Progress since Rethinking Construction and Thoughts for Our Future, London: Constructing Excellence.

[34] Akintoye, A., McIntosh, G. \& Fitzgerald, E. 2000. A survey of supply chain collaboration and management in the UK construction industry. European Journal of Purchasing and Supply Management, 6, 159-168.

[35] Cheng, E.W.L., LI, H., Love, P.E.D. 2000 Establishment of Critical Success Factors for Construction Partnering, Journal of Management in Engineering , 16(2) 84-92.

[36] Tang, W., Duffield, C.F., Young, D.M. (2006) Partnering Mechanism in Construction: an Empirical Study on the Chinese Construction Industry, Journal of Construction Engineering and Management , 132(3), 217-29.

[37] Chen, W.T., Chen, T.T. 2007. Critical Success Factors 
for Construction Partnering in Taiwan, International Journal of Project Management, 25(5), 475-84.

[38] Moore, C., Mosley, D., Slagle, M. 1992. Partnering: Guidelines for Win-Win Project Management, Project management Journal, 23(1) 18-21.

[39] Mohr, J., Spekman , R. 1994. Characteristics of Partnering Success: Partnering Attributes, Communication Behaviour and Conflict Resolution Techniques, Strategic Management Journal, 15(2) 13552.

[40] Hellard, R.B. 1996. The Partnering Philosophy - A Procurement Strategy for Satisfaction through a Team Work Solution to Project Quality, Journal of
Construction Procurement, 2(1), 41-55.

[41] Okae Adow, A-A. M.2013. Evaluating the Success Factors of Partnering in the Building Construction Industry in Accra Metropolis, Ghana, Industrial Engineering Letters www.iiste.org , ISSN 2224-6096 (Paper) ISSN 2225-0581 (online), 3(11),

[42] Bennett, J. 2000. The Seven Pillars of Partnering: a guide to second generation partnering, United Kingdom: Thomas Telford.

[43] Warfield, J.N. 1974. Developing interconnection matrices in structural modeling. IEEE Transactions on Systems, Man, and Cybernetics, (1), 81-87. 